\title{
A Reference Point Approach to Bi-Objective Dynamic Portfolio Optimization
}

\author{
Bartosz Sawik ${ }^{\star}$
}

\begin{abstract}
The portfolio selection problem presented in this paper is formulated as a biobjective mixed integer program. The portfolio selection problem considered is based on a dynamic model of investment, in which the investor buys and sells securities in successive investment periods. The problem objective is to dynamically allocate the wealth on different securities to optimize by reference point method the portfolio expected return and the probability that the return is not less than a required level. In computational experiments the dataset of daily quotations from the Warsaw Stock Exchange were used.
\end{abstract}

Keywords: Dynamic Portfolio, Mixed Integer Programming, Reference Point Method, BiObjective Optimization, Value-at-Risk

Mathematics Subject Classification: 90C11, 90C90, 91G10

Revised: 21 October 2009

\section{INTRODUCTION}

The optimal security selection is a classical portfolio problem since the seminal work of Markowitz (Markowitz, 1952, 1997). In the standard approach, the decision maker selects the securities in such a way that the portfolio expectation is maximized, under the constraint that risk (variance) must be kept under a fixed threshold (Benati, 2007, Lin, 2009). The problem consists in picking the best amount of securities, with the aim of maximizing future returns. It is a typical multivariate problem: the only way to improve future returns is to increase the risk level that the decision maker is disposed to accept (Ogryczak, 2000, Young, 1998).

In Markowitz's approach the problem is formulated as an optimization problem involving two criteria: the reward of a portfolio, which is measured by the mean and should be maximized, and the risk of the portfolio (measured by the variance of return) that should by minimized. In the presence of two criteria there is not a single optimal solution (portfolio), which represents the tradeoff between risk and return (Anagnostopoulos, 2010).

\footnotetext{
* Department of Applied Computer Science, Faculty of Management, AGH University of Science and Technology, Kraków, Poland. E-mail: b_sawik@yahoo.com
} 
While the original Markowitz model forms a quadratic programming problem, many attempts have been made to linearize the portfolio optimization procedure (Sawik, 2009a, 2009b, 2009c, 2009d, 2009e, 2009f, 2009g, 2008, Speranza, 1993, Young, 1998). The linear program solvability is very important for applications to real-life financial and other decisions where the constructed portfolios have to meet numerous side constraints. Examples of them are minimum transaction lots, transaction costs or mutual funds characteristics etc. The introduction of these features leads to mixed integer program problems. This paper presents a bi-criterion extension of the Markowitz portfolio optimization model, in which the variance has been replaced with the Value-at-Risk (VaR). The VaR is a quantile of the return distribution function (Benati, 2007, Sawik, 2009a, 2008).

The advantage of using VaR measure in portfolio optimization is that this value of risk is independent of any distribution hypothesis. It concerns only downside risk, namely the risk of loss. This index measures the loss in question in a certain way. Finally VaR is valid for all types of securities and therefore either involve the various valuation models or be independent of these models (Esch, 2005, Gaivoronski 2005).

This portfolio optimization problem is formulated as a bi-objective mixed integer program. The portfolio selection problem considered is based on a dynamic model of investment, in which the investor buys and sells securities in successive investment periods. The problem objective is to dynamically allocate the wealth on different securities to optimize by reference point method of the portfolio expected return and the probability that the return is not less than a required level.

The results of some computational experiments with the mixed integer programming approach modeled on a real data from the Warsaw Stock Exchange are reported. The input dataset consist of time series of the daily quotation of returns of securities from the Warsaw Stock Exchange.

\section{REFERENCE POINT METHOD}

The reference point method is based on the Tchebycheff metric (Alves, 2007, Bowman, 1976). Let us denote by $\|f(x)-\underline{f}\|_{\lambda}$ the $\lambda$-weighted Tchebycheff metric, i.e., $\min _{1 \leqslant l \leqslant q}\left\{\lambda_{l}\left|f_{l}(x)-\underline{f}\right|\right\}$, where $\lambda_{l} \geqslant 0 \quad \forall \sum_{l=1}^{q} \lambda_{l}=1$, and $\underline{f}$ denotes a reference point of the criteria space. Considering $f(x)>\underline{f}$ for all $x \in X$, it has been proven (Bowman, 1976) that the parametrization on $\lambda$ of $\min _{x \in X}\left\|f_{l}(x)-\underline{f}\right\|_{\lambda}$ generates the non-dominated set. The program $\min _{x \in X}\left\|f_{l}(x)-\underline{f}\right\|_{\lambda}$ may yield weakly nondominated solutions, which can be avoided by considering the augmented weighted Tchebycheff program:

$$
\begin{aligned}
\text { Minimize } \delta+\gamma \sum_{l=1}^{q} f_{l}(x) & \\
\text { subject to } \quad \lambda_{l}\left(f_{l}(x)-\underline{f}\right) & \leqslant \delta, \quad 1 \leqslant l \leqslant q \\
x & \in X, \\
\delta & \geqslant 0,
\end{aligned}
$$


where $\gamma$ is a small positive value. It has been proven (Steuer, 1986) that there always exists $\gamma$ small enough that enable to reach all the non-dominated set for the finitediscrete and polyhedral feasible region cases (Alves, 2007).

\section{PROBLEM FORMULATION}

Let $n$ be the number of securities available in the market with historical quotations in $t$ investment periods, each consisting of $h$ historical periods.

Let $r_{i j}$ be the random variable representing the future daily return of security $j$ in historical time period $i$.

The portfolio optimization problem with Value-at-Risk constraint is formulated as the classic Markowitz approach, but with Value-at-Risk instead of variance as a risk measure.

Table 1. Notation

\begin{tabular}{ll}
\hline & \multicolumn{1}{c}{ Indices } \\
\hline$i$ & $=$ historical time period $i, i=1, \ldots, m$ (i.e. day) \\
$j$ & security $j, j=1, \ldots, n$ \\
$k$ & historical successive investment period $k, k=1, \ldots, t$ (i.e. year, quarter or month, \\
& etc) \\
\hline$h \quad$ & Input parameters \\
$p_{i}=$ & probability assigned to the occurrence of past realization $i$ \\
$r_{i j}=$ & observed return of security $j$ in historical time period $i$ \\
$r^{M i n}=$ & minimum return observed in the market \\
$r^{V a R}=$ & return Value-at-Risk \\
$v$ & accepted number of securities in portfolio in each successive investment period \\
$\lambda$ & $=$ weight for the objective functions $f_{1}$ \\
$\gamma$ & small positive value \\
$f_{1}^{\text {opt }}=$ & ideal solution value of average return \\
$f_{2}^{\text {opt }}=$ & ideal solution value of average risk probability \\
\hline &
\end{tabular}


The decision maker fixes the lower bound $r^{V a R}$ for successful returns - any investments whose Value-at-Risk is less than $r^{V a R}$ will be not acceptable.

Let $r^{M i n}$ be the minimum return that can be observed in the market, for example the biggest possible loss of money invested in portfolio. In the worst case it is the whole amount of capital, so for instance it can be equal - 100\% (Benati, 2007, Lin, 2009).

The bi-objective dynamic portfolio optimization model with Value-at-Risk is an NP-hard problem even when future returns are described by discrete uniform distributions (Ehrgott, 2000, Nemhauser, 1999, Steuer, 1986).

The seven types of variables for each successive investment period are introduced in the model: a continuous wealth allocation variable that represents the percentage of wealth allocated to each security, a continuous wealth allocation variable for buying amount of each security, a continuous wealth allocation variable for selling amount of each security, a binary selection variable that prevents the choice of portfolios whose $V a R$ is below the fixed threshold and a binary selection variable for selecting each security to the portfolio and finally continuous variable represents deviation from the reference solution.

\section{OPTIMIZATION MODEL}

In the approach proposed in this paper, the portfolio optimization problem is formulated as reference point method dynamic bi-objective mixed integer program, which allows commercially available software (e.g. AMPL/CPLEX (Fourer, 1990) to be applied for solving medium size, yet practical instances. The problem formulation is presented below.

Minimize

$$
\delta+\gamma\left(-\sum_{k=1}^{t}\left(\sum_{i=(k-1) h+1}^{k h} p_{i} \sum_{j=1}^{n} r_{i j} x_{j k}\right)+\sum_{k=1}^{t} \alpha_{k}^{a V a R}\right)
$$

subject to

$$
\begin{gathered}
\lambda\left(-\sum_{k=1}^{t}\left(\sum_{i=(k-1) h+1}^{k h} p_{i} \sum_{j=1}^{n} r_{i j} x_{j k}\right)+f_{1}^{o p t}\right) \leqslant \delta \\
(1-\lambda)\left(\sum_{k=1}^{t} \alpha_{k}^{a V a R}-f_{2}^{o p t}\right) \leqslant \delta \\
y_{i k} \leqslant \frac{\sum_{j=1}^{n} r_{i j} x_{j k}-r^{\text {Min }}}{r^{\text {VaR }}-r^{\text {Min }}}, \quad i=(k-1) h+1, \ldots, k h, \quad k=1, \ldots, t \\
y_{i k}^{n} \sum_{j=1}^{n} r_{i j} x_{j k}-r^{\text {Min }} \\
r^{\text {VaR }-r^{\text {Min }}}-1, \quad i=(k-1) h+1, \ldots, k h, \quad k=1, \ldots, t
\end{gathered}
$$




$$
\sum_{i=(k-1) h+1}^{k h} p_{i}\left(1-y_{i k}\right) \leqslant \alpha_{k}^{\alpha V a R}, k=1, \ldots, t
$$

The objective function (1) represents the weighted deviation from the reference point for the portfolio expected return and the probability that the return is not less than a required level. The deviations are defined in constraints (2), (3).

Constraints (4), (5) and (6) prevent the choice of portfolios whose $V a R$ is below the fixed threshold. Every time expected portfolio return is below $r^{V a R}$, then $y_{i k}$ must be equal to 0 and $1-y_{i k}=1$ in constraint (6). Therefore, all probabilities of events $i$ whose returns are below the $V a R$ threshold was summed up. If the result is greater than $\alpha^{V a R}$, then the portfolio is not feasible.

$$
\sum_{j=1}^{n} x_{j k}=1, \quad k=1, \ldots, t
$$

Constraint (7) requires that in each investment period all capital must be allocated on different securities with positive expected return.

$$
\begin{array}{rlrl}
x_{j 1}^{\text {buy }} & =x_{j 1}, & j=1, \ldots, n: \sum_{i=1}^{h} p_{i} r_{i j}>0 \\
x_{j 1}^{\text {sell }}=0, & j=1, \ldots, n \\
x_{j k}=x_{j k-1}+x_{j k}^{\text {buy }}-x_{j k}^{\text {sell }}, & j=1, \ldots, n, \quad k=2, \ldots, t
\end{array}
$$

Constraints (8), (9) and (10) are responsible for a dynamic balance among $x_{j k}$, $x_{j k}^{b u y}, x_{j k}^{s e l l}$ for each successive investment period $k$.

$$
\sum_{j=1}^{n} z_{j k} \geqslant v, \quad k=1, \ldots, t
$$

Constraint (11) ensures that the number of stocks in optimal portfolio must be greater than or equal to the accepted number of assets in the selected portfolio.

$$
\sum_{i=(k-1) h+1}^{k h} p_{i} \sum_{j=1}^{n} r_{i j} x_{j k}>=r^{V a R}, k=1, \ldots, t
$$

Constraint (12) imposes the minimum portfolio expected return equal $r^{V a R}$ that the decision maker is prepared to accept for each successive investment period $k$.

$$
x_{j k} \leqslant z_{j k}, \quad j=1, \ldots, n: \sum_{i=(k-1) h+1}^{k h} p_{i} r_{i j}>0, k=1, \ldots, t
$$




$$
\begin{aligned}
& x_{j k}^{b u y} \leqslant z_{j k}, \quad j=1, \ldots, n: \sum_{i=(k-1) h+1}^{k h} p_{i} r_{i j}>0, k=1, \ldots, t \\
& x_{j k}^{\text {sell }} \leqslant z_{j k}, \quad j=1, \ldots, n, \quad k=1, \ldots, t
\end{aligned}
$$

Constraints (13), (14) and (15) are responsible for relations between variables $x_{j k}, x_{j k}^{b u y}, x_{j k}^{s e l l}$ and $z_{j k}$.

$$
\begin{aligned}
& \frac{z_{j k}}{100} \leqslant x_{j k}, \quad j=1, \ldots, n: \sum_{i=(k-1) h+1}^{k h} p_{i} r_{i j}>0, k=1, \ldots, t \\
& \frac{z_{j k}}{100} \leqslant x_{j k}^{b u y}, \quad j=1, \ldots, n: \sum_{i=(k-1) h+1}^{k h} p_{i} r_{i j}>0, k=1, \ldots, t
\end{aligned}
$$

Constraints (16) and (17) ensure the addition to portfolio and buying of some amount of security $\mathrm{j}$ in successive investment period $k$.

$$
0 \leqslant \alpha_{k}^{V a R} \leqslant 1, \quad k=1, \ldots, t
$$

Constraint (18) defines continuous variable $\alpha_{k}^{V a R}-$ probability that return of investment is not less than $r^{V a R}$ of successive investment period $k$.

$$
x_{j k}=0, \quad j=1, \ldots, n: \sum_{i=(k-1) h+1}^{k h} p_{i} r_{i j} \leqslant 0, k=1, \ldots, t
$$

Constraint (19) defines continuous variable $x_{j k}$ - percentage of capital invested in successive investment period $k$ in security $j$ and, in addition, eliminates securities with a non-positive expected return.

$$
\begin{aligned}
x_{j k}^{\text {buy }}=0, & j=1, \ldots, n: \sum_{i=(k-1) h+1}^{k h} p_{i} r_{i j} \leqslant 0, k=1, \ldots, t \\
x_{j k}^{\text {sell }} \geqslant 0, & j=1, \ldots, n, \quad k=1, \ldots, t \\
y_{i k} \in\{0,1\}, & i=(k-1) h+1, \ldots, k h, \quad k=1, \ldots, t \\
z_{j k} \in\{0,1\}, & j=1, \ldots, n, \quad k=1, \ldots, t \\
\delta \geqslant 0 &
\end{aligned}
$$


Finally, constraints (20)-(24) define variables $x_{j k}^{b u y}, x_{j k}^{s e l l}, z_{j k}, \delta$ and eliminate securities with a non-positive expected return. Variables $x_{j k}$ are percentage of capital invested in security $j$ in successive investment period $k$.

The combination of continuous variables $x_{j k}, x_{j k}^{b u y}, x_{j k}^{\text {sell }}$ and binary variables $y_{i k}$, $z_{j k}$ leads an NP-hard mixed integer programming problem (Nemhauser, 1999). If the number of historical observations $m$ is bounded by a constant, there are $2^{m}$ ways of fixing the variables $y_{i k}, z_{j k}$ for each successive investment period $k$.

\section{COMPUTATIONAL RESULTS}

In this section numerical examples and some computational results are presented to illustrate possible applications of the proposed formulations of this multi-period optimization model. The examples are modeled on a real data form the Warsaw Stock Exchange.

Suppose that $n$ securities with historical quotations in t investment periods, each of $h$ days, in total 4020 samples. The eighteen years horizon from 30th Jan 1991 to 30th Jan 2009 - consists of $m=4020$ historic daily quotations divided into $\mathrm{t}=20$ investment periods ( $h=201$ daily quotations each), with the selection of $n=241$ input securities for portfolio, quoted each day in the historical horizon. Probability of realization for expected securities returns is the same for each day and summed up for whole period to one. The accepted number of securities in portfolio is at least one security in each successive investment period. The basic parameters for the reference point method take on the following values: $f_{1}^{o p t}=1, f_{2}^{o p t}=0.05, \lambda=0.5, \gamma=0.01$.

The computational experiments have been performed using AMPL programming language (Fourer, 1990) and the CPLEX v.11 solver (with the default settings) on a laptop with Intel $\AA$ Core 2 Duo T9300 processor running at $2.5 \mathrm{GHz}$ and with $4 \mathrm{~GB}$ RAM. The computational time limit was set to $10800 \mathrm{CPU}$ seconds (three CPU hours).

Table 2 presents the solution results for objective function $\alpha_{k}^{V a R}$ probability that return of investment is not less than in each successive investment period $k$. Table 3 presents the solution results for the objective function of expected portfolios return in each successive investment period $k$.

Table 4 presents number of securities in the computed portfolios for each successive investment period $k$.

Table 5 presents computational time range and the solution values of $\delta$ - the deviation from the reference solution. In the table 5, column "MIP simplex iteration" shows the number of mixed integer programming simplex iterations until presented solution. Column "B-\&-B" shows the number of searched nodes in the branch and bound tree until presented solution. Column "CPU" shows CPU seconds required for proving optimality on a laptop with Intel@ Core 2 Duo T9300 processor running at $2.5 \mathrm{GHz}$ and with 4GB RAM using the solver CPLEX v.11. 


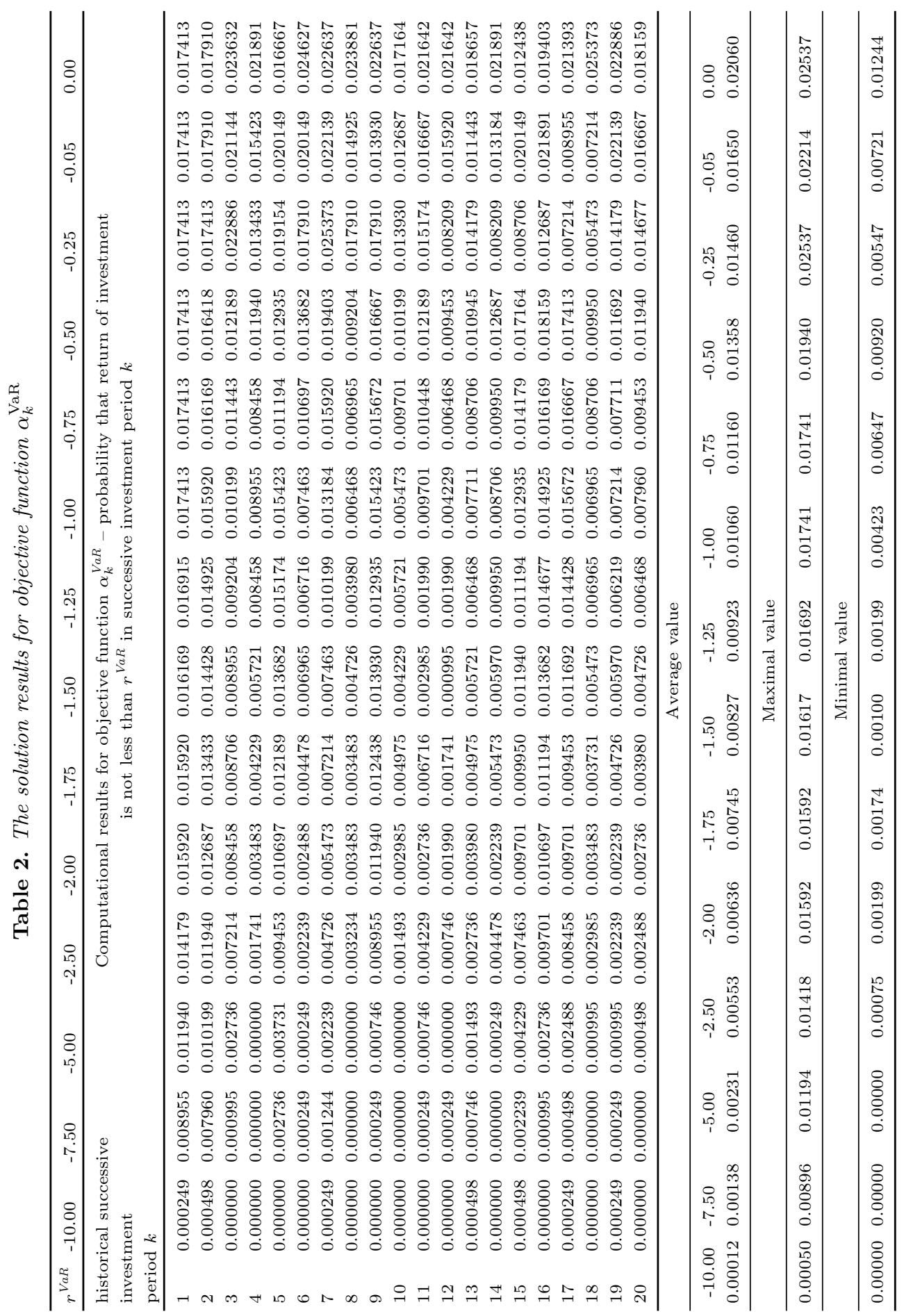




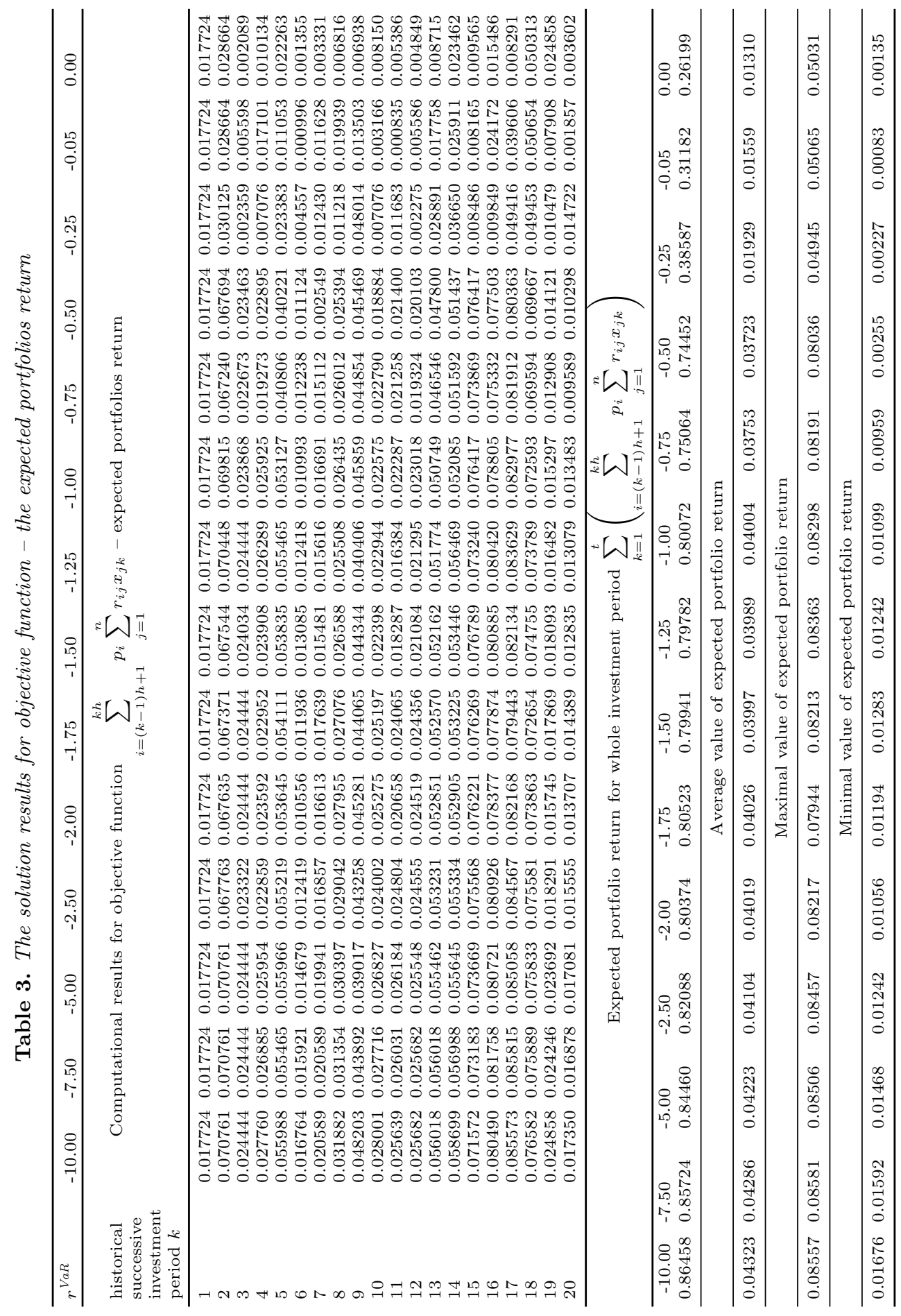


Table 4. The solution results for objective function - the expected portfolios return

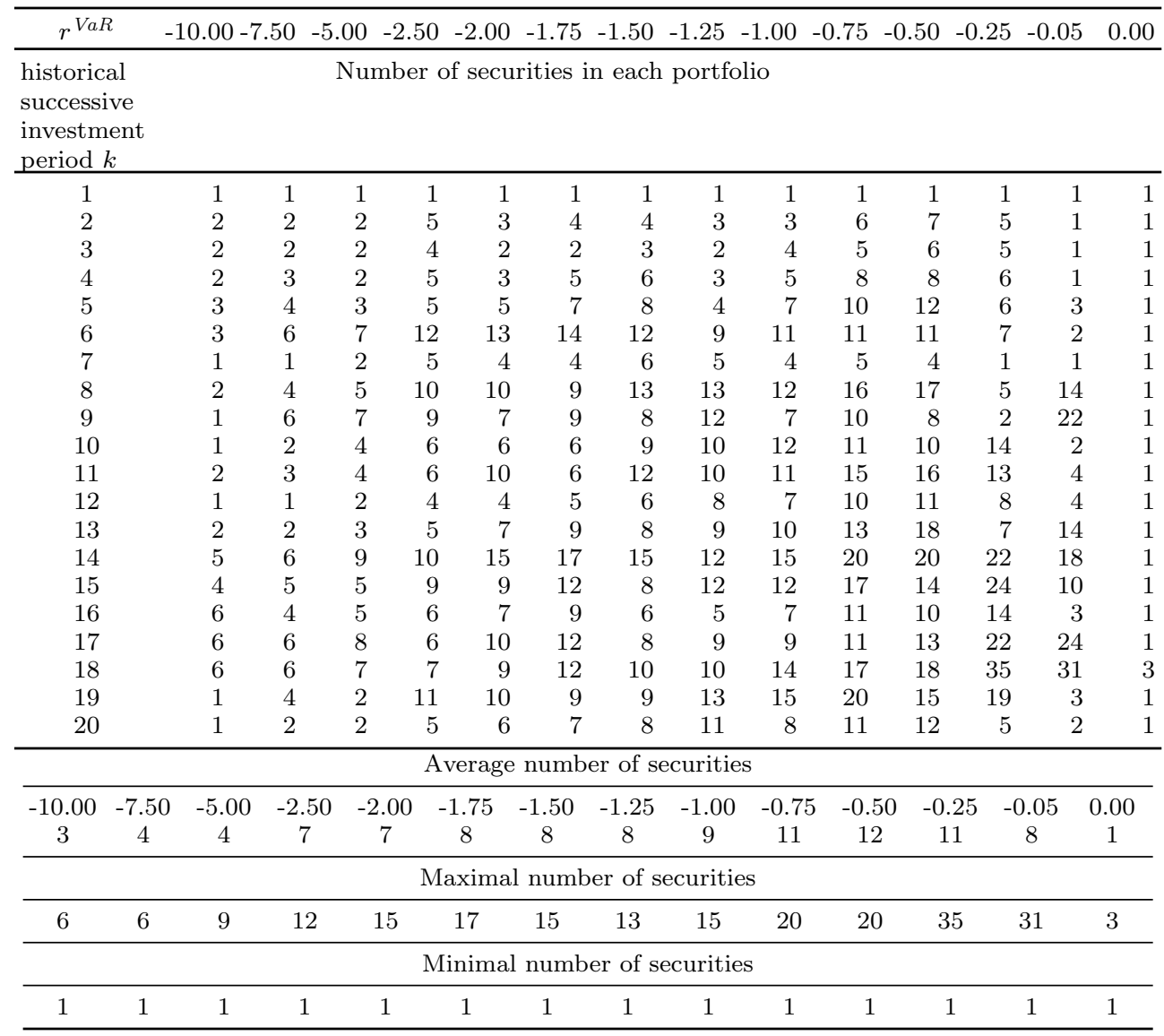

Table 5. Computational time range and the solution results for $\delta$

\begin{tabular}{rcccrr}
\hline$r^{\text {VaR }}$ & $\delta$ & $\begin{array}{c}\text { MIP simplex } \\
\text { iterations }\end{array}$ & $\begin{array}{c}\text { branch-and- } \\
\text { bound nodes }\end{array}$ & CPU & \multicolumn{1}{c}{$\begin{array}{c}\text { GAP } \\
{[\%]}\end{array}$} \\
\hline-10.00 & 0.067711 & 15,104 & 805 & 43.1 & - \\
-7.50 & 0.071381 & $3,182,319$ & 441,196 & $10,800.0$ & 1.85 \\
-5.00 & 0.077699 & $3,169,107$ & 195,210 & $10,800.1$ & 8.72 \\
-2.50 & 0.089562 & $1,668,609$ & 116,278 & $10,800.1$ & 29.31 \\
-2.00 & 0.098133 & $1,554,694$ & 76,373 & $10,800.2$ & 32.57 \\
-1.75 & 0.097387 & $1,177,463$ & 69,993 & $10,800.3$ & 36.15 \\
-1.50 & 0.100295 & $1,162,975$ & 65,026 & $10,800.3$ & 38.61 \\
-1.25 & 0.101089 & $1,713,553$ & 71,822 & $10,800.4$ & 39.98 \\
-1.00 & 0.099639 & $2,744,317$ & 95,456 & $10,800.1$ & 42.44 \\
-0.75 & 0.124678 & $2,730,531$ & 101,598 & $10,800.1$ & 47.37 \\
-0.50 & 0.127738 & $2,824,989$ & 84,196 & $10,800.4$ & 51.10 \\
-0.25 & 0.307067 & $3,022,921$ & 76,426 & $10,800.1$ & 65.44 \\
-0.05 & 0.344089 & $3,027,166$ & 71,625 & $10,800.1$ & 67.75 \\
0.00 & 0.369005 & $3,561,349$ & 84,385 & $10,800.0$ & 71.55 \\
\hline
\end{tabular}


Table 6 presents optimization problem size after presolving.

Table 6. Problem size after presolving

\begin{tabular}{|c|c|c|c|c|c|c|}
\hline \multirow[t]{2}{*}{$r^{V a R}$} & \multicolumn{4}{|c|}{ Size of adjusted problem } & \multicolumn{2}{|c|}{ Eliminated by presolving } \\
\hline & constraints & variables & $\begin{array}{c}\text { binary } \\
\text { variables }\end{array}$ & $\begin{array}{c}\text { linear } \\
\text { variables }\end{array}$ & constraints & variables \\
\hline-10.00 & 15,509 & 12,305 & 7,865 & 4,440 & 19,344 & 87,396 \\
\hline-7.50 & 15,722 & 12,416 & 7,976 & 4,440 & 19,131 & 87,285 \\
\hline-5.00 & 15,927 & 12,526 & 8,086 & 4,440 & 18,926 & 87,175 \\
\hline-2.50 & 16,180 & 12,655 & 8,215 & 4,440 & 18,673 & 87,046 \\
\hline-2.00 & 16,203 & 12,667 & 8,227 & 4,440 & 18,650 & 87,034 \\
\hline-1.75 & 16,216 & 12,674 & 8,234 & 4,440 & 18,637 & 87,027 \\
\hline-1.50 & 16,238 & 12,685 & 8,245 & 4,440 & 18,615 & 87,016 \\
\hline-1.25 & 16,261 & 12,698 & 8,258 & 4,440 & 18,592 & 87,003 \\
\hline-1.00 & 16,280 & 12,708 & 8,268 & 4,440 & 18,573 & 86,993 \\
\hline-0.75 & 16,308 & 12,722 & 8,282 & 4,440 & 18,545 & 86,979 \\
\hline-0.50 & 16,333 & 12,735 & 8,295 & 4,440 & 18,520 & 86,966 \\
\hline-0.25 & 16,353 & 12,745 & 8,305 & 4,440 & 18,500 & 86,956 \\
\hline-0.05 & 16,353 & 12,745 & 8,305 & 4,440 & 18,500 & 86,956 \\
\hline 0.00 & 16,360 & 12,866 & 8,426 & 4,440 & 18,493 & 86,835 \\
\hline
\end{tabular}

In the computational experiments the dataset of daily quotations from the Warsaw Stock Exchange were used. The computational time for the optimization model depends strongly on return Value-at-Risk parameter. The bigger value of $r^{V a R}$ is set, the more CPU seconds is required for proving optimality. The computed values of expected portfolio return and the probability that return of investment is not less than $r^{V a R}$ also depend on $r^{V a R}$. Increasing the value of $r^{V a R}$ results in decreasing of expected portfolio return and increasing of probability that the return of investment is not less than $r^{V a R}$. Number of securities in obtained portfolios varies between one and thirty five.

\section{CONCLUSIONS}

In this paper a bi-objective portfolio selection by mixed integer programming has been proposed. The considered problem is based on a dynamic model of investment, in which the investor buys and sells securities in successive investment periods. The problem objective is to dynamically allocate the wealth on different securities to optimize by reference point method the portfolio expected return and the probability that the return is not less than a required level. The model incorporates dynamic balance constraints that allow the short-selling variables to take on non-negative values.

The computational experiments modeled on a real data from the Warsaw Stock Exchange have indicated that the approach is capable of finding optimal solutions for medium size problems in a reasonable computation time using commercially available software for mixed integer programming. The total computation time ranges from minutes to hours depending on the number of historical quotations in the optimization problem. 


\section{ACKNOWLEDGEMENTS}

This work has been supported by Polish Ministry of Science and Higher Education (MNISW) grant for PhD Research \#N N519 405934.

\section{REFERENCES}

Alves, M.J., Climaco, J. (2007). A review of interactive methods for multiobjective integer and mixed-integer programming, European Journal of Operational Research, Vol. 180, pp 99-115.

Anagnostopoulos, K.P., Mamanis, G. (2010). A portfolio optimization model with three objectives and discrete variables, Computers and Operations Research, Vol. 37, pp 12851297.

Benati, S., Rizzi, R. (2007). A mixed integer linear programming formulation of the optimal mean/Value-at-Risk portfolio problem, European Journal of Operational Research, Vol. 176, pp 423-434.

Bowman Jr, V.J. (1976). On the relationship of the Tchebycheff norm and the efficient frontier of multi-criteria objectives, In: Thiriez H., Zionts S. (Eds.) Multiple Criteria Decision Making, Lecture Notes in Economics and Mathematical Systems, Vol. 130. Springer-Verlag, Berlin, Germany, pp. 76-86.

Ehrgott, M. (2000). Multicriteria Optimization. Second edition, Springer, Berlin, Germany.

Esch, L., Kieer, R., Lopez, T., Berb, C., Damel, P., Debay, M., Hannosset, J.-F. (2005). Asset and Risk Management. Risk Oriented Finance, John Wiley \& Sons.

Fourer, R., Gay, D.M., Kernighan, B.W. (1990). A Modeling Language for Mathematical Programming, Management Science, Vol. 36, pp 519-554.

Gaivoronski, A.A., Krylov, S., Van Der Wijst, N. (2005). Optimal portfolio selection and dynamic benchmark tracking, European Journal of Operational Research, Vol. 163, pp 115131.

Lin, C.C. (2009). Comments on "A mixed integer linear programming formulation of the optimal mean/Value-at-Risk portfolio problem", European Journal of Operational Research, Vol. 194, pp 339-341.

Markowitz, H.M. (1952). Portfolio selection, Journal of Finance, Vol. 7, pp 77-91

Markowitz, H.M. (1997). Portfolio Selection: Efficient Diversification of Investments. Second edition, Blackwell Publishers, Inc., Malden, Mass., USA.

Nemhauser, G.L., Wolsey, L.A. (1999). Integer and Combinatorial Optimization, John Wiley \& Sons, Toronto, Canada.

Ogryczak, W. (2000). Multiple criteria linear programming model for portfolio selection, Annals of Operations Research Vol. 97, pp 143-16.

Sawik, B. (2009a). Lexicographic and Weighting Approach to Multi-Criteria Portfolio Optimization by Mixed Integer Programming, In: Lawrence K. D., Kleinman G. (Eds.) Applications of Management Science, Vol. 13, Financial Modeling Applications and Data Envelopment Applications, Emerald Group Publishing Limited, UK, USA, pp. $3-18$.

Sawik, B. (2009b). A Multi-Objective Dynamic Portfolio Optimization with Short Selling Variables, INFORMS annual meeting, Oct 11-14, 2009, San Diego, USA.

Sawik, B. (2009c). A Weighted-Sum Mixed Integer Program for Bi-Objective Dynamic Portfolio Optimization, Semi-Annual "Automatyka" Vol. 13(2), pp 563-571. 
Sawik, B. (2009d). A Lexicographic Approach for Multi-Objective Dynamic Portfolio Optimization, The 23rd European Conference on Operational Research (EURO XXIII), Jul 5-8, 2009, Bonn, Germany.

Sawik, B. (2009e.) Portfolio Optimization of a Multi-Period Investment by Mixed Integer Programming, In: Howaniec H., Waszkielewicz W. (Eds.) Conditions of development of management systems, Monographic of ATH University of Bielsko-Biała, Poland, pp. $112-120$.

Sawik, B. (2009f). A Dynamic MIP Approach to Multi-Objective Portfolio Optimization, CORS-INFORMS, Jun 14-17, 2009, Toronto, Canada.

Sawik, B. (2009g). Bi-Objective Dynamic Portfolio Optimization by Mixed Integer Programming, European Chapter on Combinatorial Optimization (ECCO XXII), May 17-20, 2009, Jerusalem, Israel.

Sawik, B. (2008). A Three Stage Lexicographic Approach for Multi-Criteria Portfolio Optimization by Mixed Integer Programming, Przeglad Elektrotechniczny, Vol. 84(9), pp 108-112.

Speranza, M.G. (1993). Linear programming models for portfolio optimization, Finance, Vol. 14, pp 107-123.

Steuer, R.E. (1986). Multiple Criteria Optimization: Theory, Computation and Application, John Wiley \& Sons, New York, USA.

Young, M.R. (1998). A minimax portfolio selection rule with linear programming solution, Management Science, Vol. 44, pp 673-683. 